\title{
The Bivariate Model of Double-line Method- based Moistening Deformation Coefficient
}

\author{
http://dx.doi.org/10.3991/ijoe.v11i8.4877 \\ Xi Chen , Hong Guo \\ Shaanxi University of Technology, Hanzhong, Shaanxi, China
}

\begin{abstract}
The paper analyzes the express method of doubleline method-based moistening deformation coefficient, which can be expressed as the strain difference with same pressure and different water contents. Through the analysis it is found that the key point of constructing moistening deformation coefficient bivariate model is to obtain the function relation of strain, stress and water content. And then, the paper discusses the concept of constructing moistening deformation coefficient bivariate model under confining compression test conditions. In combination with the specific test shows the bivariate model of moistening deformation coefficient realizing process. Finally, the verification test results show that the model is reasonable.
\end{abstract}

Index Terms - moistening deformation coefficient, model, method

\section{INTRODUCTION}

Loess is a kind of Quaternary-sediments, mainly distributed in arid and semiarid regions. Loess has a series of internal composition and the external morphological characteristics due to the generation of specific environment, which is of silt, low density, vertical joints development, high soluble salt content and so on [1-4].

The large area in the northwest of china and the middle reaches of yellow river is covered with loess. The loess has different collapsibility due to its special engineering properties and structure characteristics. Because of its direct relation to the construction cost and safety, it has been an important issue in the field of geotechnical engineering. In recent years, with in-depth study of loess collapsibility, a large number of research results have been obtained [5-11]. The research emphasis of the loess collapsibility is gradually transferred from collapsible deformation to moistening deformation. Moistening deformation contains all deformation generated by humidification under pressure, the definition domain of which is wider than that of collapsible deformation. It is also called generalized collapsibility[6-8]. Water and loading are important conditions for loess moistening deformation. Therefore, the research on the effect of water and loading on loess collapsibility is one of the important subject of loess collapsibility.

This paper mainly discussed the basic method of constructing bivariate model considering moisture content and stress. Also combined with the specific test, it shows the realization process of the method and test the constructed model.
II. THE CONCEPT OF CONSTRUCTING DOUBLE-LINE METHOD-DASED MOISTENING DEFORMATION COEFFICIENT BIVARIATE MODEL UNDER CONFINING COMPRESSION TEST

The moistening deformation is refers to the additional settlement after the collapsible loess sinking stability under a certain pressure. The moistening deformation per thickness of soil samples is defined as moistening deformation coefficient [6]. Its expression is:

$$
\delta_{s}^{\prime}=\frac{h_{p}-h_{p}^{\prime}}{h_{0}}
$$

Where $\delta_{s}^{\prime}$ is moistening deformation coefficient; $h_{p}$ is the height of soil after sinking stability under the action of pressure $p$ when the initial moisture content is $w_{0} ; h_{p}^{\prime}$ is the height of soil after sinking stability under the action of pressure $p$ and moistening to moisture content $w ; h_{0}$ is the soil original height.

Usually, there are two research approaches in laboratory test for collapsible loess, namely, single line method and double line method. The double line method is widely used at present because of its briefness, less workload, strong comparative and so on. When the double line method is used to test moistening deformation coefficient, the type 1 can be written as:

$$
\delta_{s}^{\prime}=\frac{h_{p}-h_{p}^{\prime}}{h_{0}}=\frac{\left(h_{0}-h_{p}^{\prime}\right)-\left(h_{0}-h_{p}\right)}{h_{0}}=\varepsilon_{w}-\varepsilon_{0}
$$

Where $\varepsilon_{w}$ is soil strain under the pressure $p$ when the water content is $w ;{ }^{\varepsilon_{0}}$ is soil strain under the pressure $p$ when the initial water content is $w_{0}$. That is the doubleline method-dased moistening deformation coefficient can be expressed as the strain difference with same pressure and different water contents.

As shown in (2), the key point of constructing moistening deformation coefficient bivariate model is to obtain the function relation of strain, stress and moisture content $(\varepsilon=f(\sigma, w))$.

The typical relationship curve of soil strain - stress under confining compression test is shown in Figure 1. 


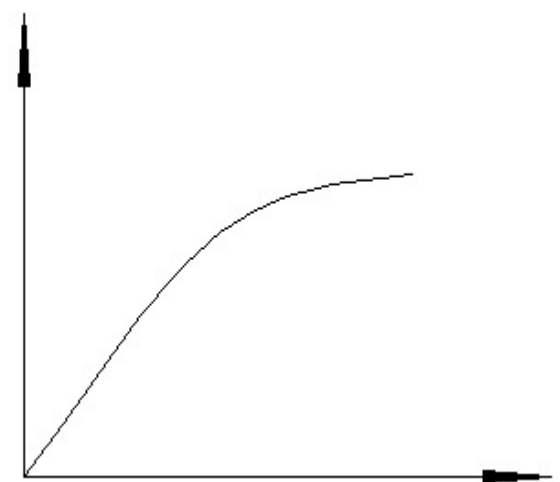

Figure 1. The typical relationship curve of soil strain - stress under confining compression test

As is shown in the Figure 1, the relationship curve of soil strain - stress under confining compression test is hyperbolic, its expression can be shown as in (3):

$$
\varepsilon=\frac{\sigma}{a+b \sigma}
$$

Where $a$ and $b$ are the test parameters.

In (3), the influence of moisture content on the soil strain - stress relationship under confining compression test can be shown in the test parameters $a$ and $b$ .Therefore, as long as the influence of moisture content on relationship of soil strain - stress separated from the parameters $a$ and $b$, the function relation of strain and stress, and moisture content can be established.

If the test parameters are $a$ and $b$ under confining compression test when the moisture content is $\mathrm{w}$; the test parameters are $a_{s}$ and $b_{s}$ when the soil is saturated. The water content and the ratio of test parameter $a$ and $a_{s}$ have a

functional relationship. It can be written as ( $a_{s}$

$$
\frac{a}{a}=j(w)
$$

). Likewise, The water content and the ratio of test parameter $b$ and $b_{s}$ also have a functional relationship. It can be written as $\left(\frac{b}{b_{s}}=g(w)\right.$.

then :

$$
\begin{aligned}
& a=a_{s} \frac{a}{a_{s}}=a_{s} j(w) \\
& b=b_{s} \frac{b}{b_{s}}=b_{s} \& \cdot
\end{aligned}
$$

So the general relational expression of soil strain stress can be written as:

$$
\varepsilon=\frac{\sigma}{a_{s} j(w)+b_{s} g(w) \sigma}
$$

This proves that once the concrete expression of $\frac{a}{a_{s}}=f(w) \quad \frac{b}{b_{s}}=g(w)$ can be determined. By substitution riate model of double-line method-dased to (2), the bivaformation coefficient can be established as follows:

$$
\begin{aligned}
& \delta_{s}^{\prime}=\varepsilon_{w}-\varepsilon_{0} \\
& =\frac{\sigma}{a_{s} j(w)+b_{s} g(w) \sigma}-\frac{\sigma}{a_{s} j\left(w_{0}\right)+b_{s} g\left(w_{0}\right) \sigma}
\end{aligned}
$$

Then this paper shows the bivariate model considering moisture content and stress of moistening deformation coefficient realizing process in combination with the specific test.

\section{THE CONSTRUCTION OF BIVARIATE MODEL}

\section{A. Test method and test data}

The soil samples used in the test are from Yangling City in Shannxi Province and Lanzhou City in Gansu Province. The soil samples of Yangling are taken from $3 \mathrm{~m}$ depth of second terrace of Wei River. Its natural moisture content is $19 \%$ obtained by drying measured. Its dry density is $1.28 \mathrm{~g} / \mathrm{cm}^{3}$ and saturated moisture content is $41 \%$.The soil samples of Lanzhou are taken from $5 \mathrm{~m}$ depth of YuZhong university town. Its natural moisture content is $5 \%$ obtained by drying measured. Its dry density is $1.4 \mathrm{~g} / \mathrm{cm}^{3}$ and saturated moisture content is $35 \%$. The test equipment is Triple consolidation apparatus. Using dripping with water make the moisture content of Yangling loess samples to $5 \%, 12 \%, 19 \%, 26 \%, 33 \%$ and $41 \%$ and the moisture content of Lanzhou loess samples to $5 \%, 12 \%, 19 \%, 26 \%$ and $35 \%$. Test data is shown in Figure 2.

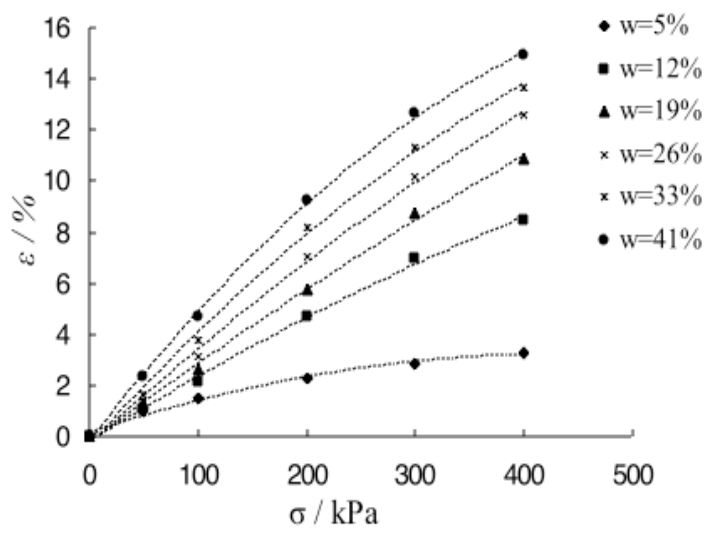

(a) Yangling loess

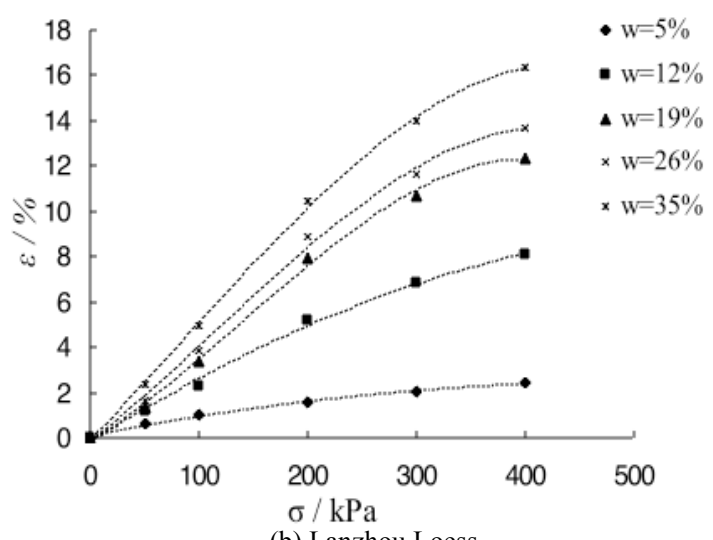

(b) Lanzhou Loess

Figure 2. The curves of stress and strain with different loess

\section{B. The construction of the model}

It can be seen from figure 2 that the relationship of soil strain -stress of Yangling and Lanzhou are a hyperbolic 
curve under confining compression test. The fitting results are shown in table 1 obtained by (3) and the experimental data.

TABLE I.

THE TEST PARAMETERS OF STRAIN -STRESS RELATIONSHIP CURVE WITH DIFFERENT LOESS

\begin{tabular}{|c|c|c|c|c|}
\hline \multirow{3}{*}{ Soil types } & \multirow{2}{*}{ Water content/\% } & \multicolumn{3}{|c|}{ fitting parameters } \\
\cline { 2 - 5 } & & $\boldsymbol{a}$ & $\boldsymbol{b}$ & $\boldsymbol{R}^{\mathbf{2}}$ \\
\hline \multirow{4}{*}{$\begin{array}{c}\text { Yangling } \\
\text { loess }\end{array}$} & 5 & 6447.80 & 12.18 & 0.99 \\
\cline { 2 - 5 } & 12 & 4252.42 & 0.89 & 1.00 \\
\cline { 2 - 5 } & 19 & 3156.80 & 1.47 & 1.00 \\
\cline { 2 - 5 } & 26 & 2538.02 & 1.83 & 1.00 \\
\cline { 2 - 5 } & 33 & 2144.71 & 2.19 & 1.00 \\
\cline { 2 - 5 } & 41 & 1761.49 & 2.58 & 0.99 \\
\cline { 2 - 5 } & 5 & 7761.37 & 22.62 & 0.99 \\
\cline { 2 - 5 } Loess & 12 & 3403.84 & 3.63 & 0.96 \\
\cline { 2 - 5 } & 18 & 2318.13 & 2.03 & 0.97 \\
\cline { 2 - 5 } & 26 & 2045.48 & 2.00 & 1.00 \\
\hline \multirow{3}{*}{\begin{tabular}{c} 
Lanzhou \\
\cline { 2 - 5 }
\end{tabular}} & 35 & 1644.79 & 1.87 & 0.98 \\
\hline
\end{tabular}

Through analysing table 1 it is can be found the parameters $a$ decreases with the increased moisture content. But the parameters $b$ of Lanzhou loess and Yangling loess are different: the parameters $b$ of Yangling loess increase firstly and then decrease with the increased moisture content; the parameters $b$ of Yangling loess decrease gradually with the increased moisture content.

In order to obtain the concrete expression of $\frac{a}{a_{s}}=j(w) \quad \frac{b}{b_{s}}=g(w) \frac{a}{a_{s}}$ and $\frac{b}{b_{s}}$ should be calculated firstly. And then the curve of $\frac{a}{a_{s}}-w$ $\frac{b}{b_{s}}-w$ are drawn in Figure 3.

It can be seen from the Figure 3 that the relationship curves of $\frac{a}{a_{s}}-w$ and $\frac{b}{b_{s}}-w \quad$ can be expressed with the secondary fitting of Bertalanff curve, its expression is $y=A\left(1-B e^{-C x}\right)^{2}$. The fitting parameters are shown in Table II.

According to this, the bivariate model of the relationship of soil strain - stress of Yangling and Lanzhou, as shown in (8).

$$
\varepsilon=\frac{\sigma}{a_{s} A\left(1-B e^{-C w}\right)^{2}+b_{s} A_{1}\left(1-B_{1} e^{-C_{1} w}\right)^{2} \sigma}
$$

For the loess in Yangling :

$$
\varepsilon=\frac{\sigma}{1444.42\left(1+1.50 e^{-6.032 w}\right)^{2}+2.12\left(1-15 e^{-29.69 w}\right)^{2} \sigma}
$$

For the loess in Lanzhou :

$$
\varepsilon=\frac{\sigma}{1430.97\left(1+3.97 e^{-11.53 w}\right)^{2}+1.87\left(1-14 e^{-33.34 w}\right)^{2} \sigma}
$$

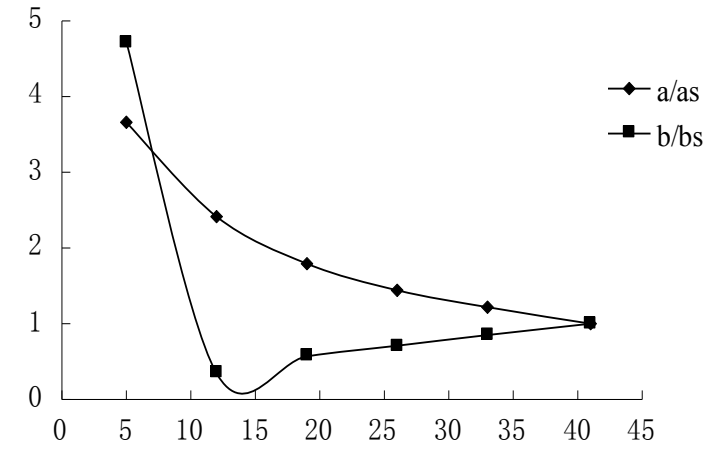

(a) Yangling loess

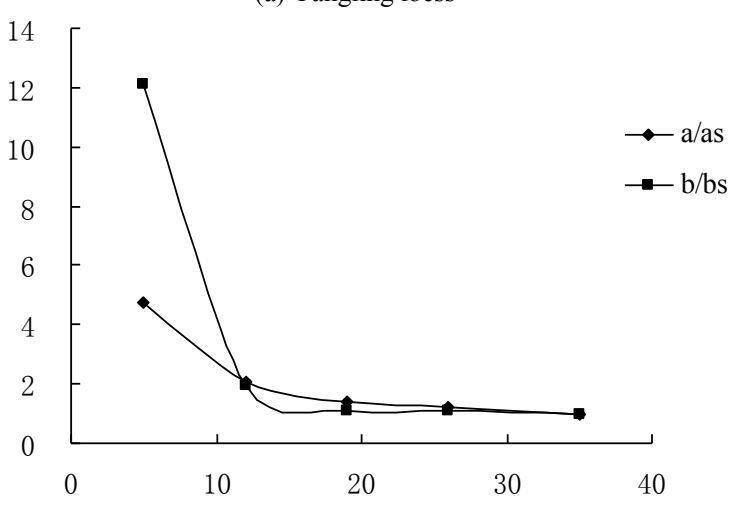

(b) Lanzhou loess Figure 3. The curve of $\frac{a}{a_{s}}-w$ and $\frac{b}{b_{s}}-w$

TABLE II.

THE LIST OF FITTING PARAMETERS

$$
\text { (a) } \frac{a}{a_{s}}
$$

\begin{tabular}{|c|c|c|c|c|}
\hline \multirow{2}{*}{ Soil types } & \multicolumn{4}{|c|}{ Fitting parameters } \\
\cline { 2 - 5 } & $A$ & $B$ & $C$ & $R^{2}$ \\
\hline Yangling loess & 0.82 & -1.50 & 6.03 & 0.99 \\
\hline Lanzhou loess & 0.87 & -3.97 & 11.53 & 1.00 \\
\hline \multicolumn{5}{|c|}{ (b) b $_{S}$} \\
\begin{tabular}{|c|c|c|c|c|}
\hline \multicolumn{5}{|c|}{ Fitting parameters } \\
\hline Soil types & $A$ & $B$ & $C$ & $R^{2}$ \\
\cline { 2 - 5 } & 0.82 & 15.00 & 29.69 & 1.00 \\
\hline Yangling loess & 1.00 & 14.00 & 33.34 & 1.00 \\
\hline Lanzhou loess & & & \\
\hline
\end{tabular}
\end{tabular}

The moistening deformation coefficient can be obtained by substituting (8) into (7), that is :

$$
\begin{aligned}
& \delta_{s}^{\prime}=\varepsilon_{w}-\varepsilon_{0} \\
& =\frac{\sigma}{a_{s} A\left(1-B e^{-C w}\right)^{2}+b_{s} A_{1}\left(1-B e^{-C_{1} w}\right)^{2} \sigma} \\
& -\frac{\sigma}{a_{s} A\left(1-B e^{-C w_{0}}\right)^{2}+b_{s} A_{1}\left(1-B e^{-C_{1} w_{0}}\right)^{2} \sigma}
\end{aligned}
$$

And then the soil moistening deformation coefficient of Yangling can be expressed in (12): 


$$
\begin{aligned}
& \delta_{s}^{\prime}=\varepsilon_{w}-\varepsilon_{0} \\
& =\frac{\sigma}{1644.79 \times 0.87\left(1+3.97 e^{-11.53 w}\right)^{2}+1.87\left(1-14 e^{-33.34 w}\right)^{2} \sigma} \\
& -\frac{\sigma}{1644.79 \times 0.87\left(1+3.97 e^{-11.53 w_{0}}\right)^{2}+1.87\left(1-14 e^{-33.34 w_{0}}\right)^{2} \sigma}
\end{aligned}
$$

The soil moistening deformation coefficient of Lanzhou

$$
\begin{aligned}
& \delta_{s}^{\prime}=\varepsilon_{w}-\varepsilon_{0} \text { be expressed in } \\
& =\frac{\sigma}{1430.97\left(1+3.97 e^{-11.53 w}\right)^{2}+1.87\left(1-14 e^{-33.34 w}\right)^{2} \sigma} \\
& -\frac{\sigma}{1430.97\left(1+3.97 e^{-11.53 w_{0}}\right)^{2}+1.87\left(1-14 e^{-33.34 w_{0}}\right)^{2} \sigma}
\end{aligned}
$$

\section{Model test}

The test data was compared with the calculated data for examining the rationality of the model. The soil moistening deformation coefficient of Lanzhou and Yangling are calculated separately with natural water content for the initial water content. The results of comparison were shown in Figure.4.

In figure 4 , the character of "**" indicates the test data. The calculated surface was set a translucent surface in order to conveniently analyze the relationship between test data and calculated data. If the test data is larger than calculation data, the character of "*" is above the calculated surface and can be clearly seen in the figure; on the other hand, the character of "*" is below the calculated surface and can be blurrily seen in the figure. It is can be seen in the figure 4 that the changing trend of connective line of calculated surface and test data are almost consistent. The test point data distribute in a small range on both sides of calculated surface. And the calculated data ties in with the test data. This shows that bivariate model of soil moistening deformation coefficient considering stress and moisture content is reasonable.

\section{CONSLUSION}

The soil moistening deformation coefficient obtained by the double line method can be expressed the strain difference with same pressure and different water contents. Through the analysis it is found that the key point of constructing moistening deformation coefficient bivariate model is to obtain the function relation of strain, stress and water content. The specific expression of soil moistening deformation coefficient bivariate model is established by the data obtained under confining compression test in Yangling loess and Lanzhou loess. The verification test for the model shows that the soil moistening deformation coefficient bivariate model is reasonable.

\section{REFERENCES}

[1] D. Z. Liu. Loess mechanics and engineering. Xi'an: Shaanxi Science and Technology Press, 1997, pp 1-10.

[2] A. Q. Gu. "Chinese loess research and its engineering application," in Geotechnical engineering retrospect and prospect. D. Z. Gao and J. Sun, Eds. Beijing: China Communications Press,2000, pp. $296-312$

[3] L. S. Zhang. "Discussions on test methods of collapsible loess," Rock and Soil Mechanics, vol.22, pp. 207-210, June 2001.

[4] W. Zhang. "The problems of loess mechanics properties test," Geotechnical Investigation \& Surveying, pp.6-12, 1995.

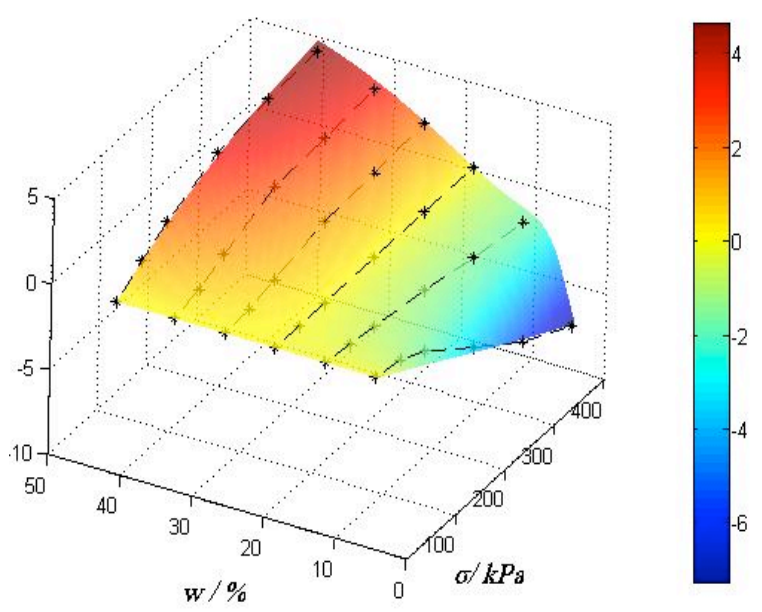

(a) Yangling loess

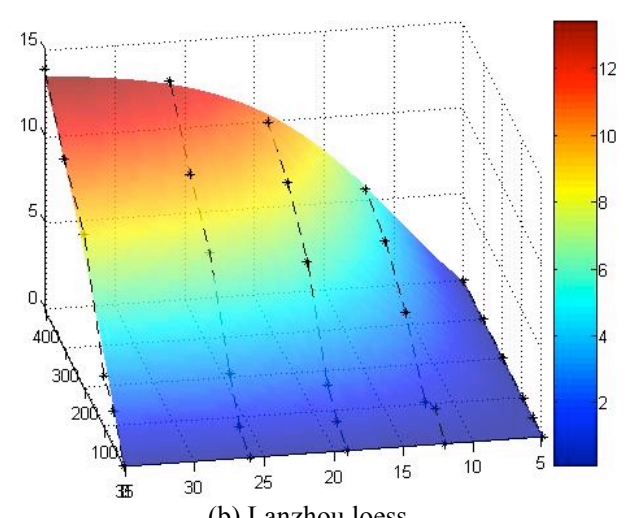

(b) Lanzhou loess

Figure 4. The compare Test data and calculated data

[5] W. Zhang, S. M. Zhang. "Development of loess engineering properties research in China," Chinese Journal of Geotechnical Engineering, vol.17, pp.80-88, November 1995.

[6] S. M. Zhang. "Terminologies and basic concepts of subsidence loess," Geotechnical Engineering Technique, pp. 42-46, 2000.

[7] S. M. Zhang, J. G. Zheng. "The deformation characteristics of collapsible loess during moistening process," Chinese Journal of Geotechnical Engineering, vol.12, pp.21-23, July 1990.

[8] S. M. Zhang, W. Zhang. "Dehumidifying and humidifying of the loess collapsibility," Chinese Journal of Geotechnical Engineering, vol.14, pp.57-61, January 1992.

[9] Y. D. Zhang. "The collapsible loess of sensitivity," Chinese Journal of Geotechnical Engineering, vol.18, pp.79-83, September 1996.

[10] Z. D. Liu, F. N. Dang, Z. Q. Hu. "The improved calculation method of collapsibility in loess foundation," Geotechnical Engineering Technique, pp. 138-141, 2001.

[11] [11] M. H. Zhang, Y. L. Xie, B. J. Liu. "Characteristics of collapsibility coefficient curves of loess during moistening and remoistening process," Rock and Soil Mechanics, vol.26, pp. 1363-1368, September 2005.

\section{AUTHORS}

X. Chen is with the School of Civil Engineering and Architecture, Shaanxi University of Technology, Hanzhong, Shaanxi, CO 723001 China (e-mail: 616137105@ qq.com).

H. Guo is with the School of Civil Engineering and Architecture, Shaanxi University of Technology, Hanzhong, Shaanxi, CO 723001 China (e-mail: aaronkwo@qq.com).

Submitted 21 July 2015. Published as resubmitted by the authors 10 October 2015. 